\title{
ORDER EQUALITIES IN DIFFERENT METRICS FOR MODULI OF SMOOTHNESS OF VARIOUS ORDERS
}

\author{
Niyazi A. Il'yasov \\ Baku State University, Baku, AZ 1148, Azerbaijan \\ niyazi.ilyasov@gmail.com
}

\begin{abstract}
In this paper, we obtain order equalities for the $k$ th order $L_{q}(T)$-moduli of smoothness $\omega_{k}(f ; \delta)_{q}$ in terms of expressions that contain the $l$ th order $L_{p}(T)$-moduli of smoothness $\omega_{l}(f ; \delta)_{p}$ on the class of periodic functions $f \in L_{p}(T)$ with monotonically decreasing Fourier coefficients, where $1<p<q<\infty, k, l \in \mathbb{N}$, and $T=(-\pi, \pi]$.
\end{abstract}

Keywords: Inequalities of different metrics for moduli of smoothness, Order equality, Trigonometric Fourier series with monotone coefficients. norm

Let $L_{p}(\mathbb{T}), 1 \leq p<\infty$, be the space of all measurable $2 \pi$-periodic functions with finite $L_{p}(\mathbb{T})$ -

$$
\|f\|_{p}=\left(\pi^{-1} \int_{\mathbb{T}}|f(x)|^{p} d x\right)^{1 / p}
$$

where $\mathbb{T}=(-\pi, \pi]$; let $E_{n}(f)_{p}$ be the best approximation of a function $f$ in the metric $L_{p}(\mathbb{T})$ by trigonometric polynomials of order at most $n, n \in \mathbb{Z}_{+}$; and let $\omega_{l}(f ; \delta)_{p}$, where $l \in \mathbb{N}$ and $\delta \in[0,+\infty)$, be the $l$ th order modulus of smoothness of a function $f \in L_{p}(\mathbb{T})$ :

$$
\omega_{l}(f ; \delta)_{p}=\sup \left\{\left\|\Delta_{h}^{l} f(\cdot)\right\|_{p}: h \in \mathbb{R},|h| \leq \delta\right\},
$$

where

$$
\Delta_{h}^{l} f(x)=\sum_{\nu=0}^{l}(-1)^{l-\nu}\left(\begin{array}{l}
l \\
\nu
\end{array}\right) f(x+\nu h), \quad\left(\begin{array}{l}
l \\
\nu
\end{array}\right)=\frac{l !}{\nu !(l-\nu) !}, \quad \nu=\overline{0, l} .
$$

The following statement contains known upper estimates for $\omega_{k}(f ; \delta)_{q}$ in terms of $\omega_{l}(f ; \delta)_{p}$, where $f \in L_{p}(\mathbb{T}), p<q$, and $l, k \in \mathbb{N}$ (see, for example, [3, Theorem 1]; the background and the corresponding references can also be found in [3]).

Theorem A. Let $1 \leq p<q<\infty, f \in L_{p}(\mathbb{T}), \sigma=1 / p-1 / q, l, k \in \mathbb{N}$, and let

$$
\Omega_{l}(f ; p ; \sigma ; q) \equiv\left(\sum_{\nu=1}^{\infty} \nu^{q \sigma-1} \omega_{l}^{q}\left(f ; \frac{\pi}{\nu}\right)_{p}\right)^{1 / q}<\infty .
$$

Then $f \in L_{q}(\mathbb{T})$ and the following estimates hold:

$$
\begin{gathered}
\|f\|_{q} \leq C_{1}(l, p, q)\left\{\|f\|_{1}+\Omega_{l}(f ; p ; \sigma ; q)\right\} \\
\omega_{k}\left(f ; \frac{\pi}{n}\right)_{q} \leq C_{2}(k, l, p, q)\left(\sum_{\nu=n+1}^{\infty} \nu^{q \sigma-1} \omega_{l}^{q}\left(f ; \frac{\pi}{\nu}\right)_{p}\right)^{1 / q}, \quad n \in \mathbb{N}, \quad l \leq k
\end{gathered}
$$




$$
\begin{aligned}
& \omega_{k}\left(f ; \frac{\pi}{n}\right)_{q} \leq C_{3}(k, l, p, q)\left\{\left(\sum_{\nu=n+1}^{\infty} \nu^{q \sigma-1} \omega_{l}^{q}\left(f ; \frac{\pi}{\nu}\right)_{p}\right)^{1 / q}+\right. \\
& \left.+n^{-k}\left(\sum_{\nu=1}^{n} \nu^{q(k+\sigma)-1} \omega_{l}^{q}\left(f ; \frac{\pi}{\nu}\right)_{p}\right)^{1 / q}\right\}, \quad n \in \mathbb{N}, \quad l>k .
\end{aligned}
$$

Hereinafter, $C_{j}(k, l, p, q, \ldots)$, where $j \in \mathbb{N}$, stand for positive values depending only on the parameters given in parentheses.

Remark 1. In addition to the background outlined in [3], certain facts the author has learned after the publication of [3] should be mentioned, which partly provide more detailed information about the situation in the matter under consideration.

1) The first part of Theorem $A: \Omega_{l}(f ; p ; \sigma ; q)<\infty \Rightarrow f \in L_{q}(\mathbb{T})$ and estimate (2) for $l=1$ were established by Ul'yanov [11, §3, Theorem 1 , statement c, inequalities (3.6)].

2) The problem of establishing estimates of type (2) by methods different from those applied in [11] was also considered by Timan $[9 ; 10$, Theorem A]. In [10, first indention after the statement of Theorem A], it was noted that the first part of Theorem A above with estimate (2) (under the assumption that $\int_{\mathbb{T}} f(x) d x=0$, which ensures the absence of the term $\|f\|_{1}$ on the right-hand side of (2); see [10, inequality (1.12)]) for $1<p<q \leq 2$ was obtained in [8]. Actually, [8] (see [8, Theorem 8]) does not contain estimate (2); instead, there were announced an assertion that leads to the implication $\Omega_{l}(f ; p ; \sigma ; \theta)<\infty \Rightarrow f \in L_{q}(\mathbb{T})$, where $\theta=\min \{2, p\}=p<q$.

3) Estimate (2) in various forms has also been obtained earlier by other authors (see, for example, [7, Introduction] and the references therein).

4) Estimate (3) for $l=k=1$ was proved by Ul'yanov [12, §4, Theorem 4, inequality (4.4)] (its formulation was given earlier in $\left[11, \S 3\right.$, second inequality in $\left.\left(3.6^{\prime}\right)\right]$, and the validity of this estimate for $l=k>1$ was also mentioned there).

5) Estimate (3) follows immediately from inequality (2) (see, for example, [7, Sect. 4], where this fact was noted for the case $l=k=1$ ). In the general case $l \leq k$, it is sufficient to apply (2) to the function $\Delta_{h}^{k} f(x)$, where $h \in \mathbb{R},|h| \leq \pi / n$, and take into account the estimates $\omega_{l}\left(\Delta_{h}^{k} f ; \pi / \nu\right)_{p} \leq$ $2^{k} \omega_{l}(f ; \pi / n)_{p}$ for $\nu \leq n$ and $\omega_{l}\left(\Delta_{h}^{k} f ; \pi / \nu\right)_{p} \leq 2^{k} \omega_{l}(f ; \pi / \nu)_{p}$ for $\nu \geq n+1$.

6) In $[8$, Theorem 8 , inequality (40)], it was announced an inequality from which estimate (4) (with an additional term of order $O\left(n^{-k}\right)$ on the right-hand side) can be obtained with $\theta=$ $\min \{2, p\}<q$ instead of $q$ on the right-hand side of this estimate.

Estimate (3) can be strengthened in the case $p>1$; more exactly, the following theorem holds.

Theorem B. Suppose that $1<p<q<\infty, f \in L_{p}(\mathbb{T}), \sigma=1 / p-1 / q, l, k \in \mathbb{N}, l \leq k$, and condition (1) holds. Then, the following estimate is valid:

$$
n^{\sigma-l}\left(\sum_{\nu=1}^{n} \nu^{p(l-\sigma)-1} \omega_{k}^{p}\left(f ; \frac{\pi}{\nu}\right)_{q}\right)^{1 / p} \leq C_{4}(k, l, p, q)\left(\sum_{\nu=n+1}^{\infty} \nu^{q \sigma-1} \omega_{l}^{q}\left(f ; \frac{\pi}{\nu}\right)_{p}\right)^{1 / q} .
$$

Estimate (5) was first obtained by Kolyada [7, Sect. 3, Theorem 2, inequality (3.8)] for the case $l=k=1$; its validity for $l=k>1$ was noted by Goldman [2, Sect. 4, proof of Lemma 6, inequality (11)]. Estimate (5) for $k>l$ follows from the well-known order equality

$$
\sum_{\nu=1}^{n} \nu^{\alpha \beta-1} \omega_{k}^{\alpha}(f ; \pi / \nu)_{q} \asymp \sum_{\nu=1}^{n} \nu^{\alpha \beta-1} \omega_{l}^{\alpha}\left(f ; \frac{\pi}{\nu}\right)_{q}, \quad n \in \mathbb{N} \cup\{+\infty\},
$$

where $1 \leq \alpha<\infty$ and $0<\beta<\min \{k, l\}$. 
Recall that an order equality $\varphi_{n} \asymp \psi_{n}$ means that there exist numbers $0<C_{5} \leq C_{6}$ depending only on the parameters given (in this case, on $k, l, \beta$, and $\alpha$ ) such that $C_{5} \psi_{n} \leq \varphi_{n} \leq C_{6} \psi_{n}$.

For given $p \in[1, \infty)$, denote by $M_{p}(\mathbb{T})$ the class of all functions $f \in L_{p}(\mathbb{T})$ whose Fourier coefficients satisfy the conditions $a_{0}(f)=0$ and $a_{n}(f) \downarrow 0$ and $b_{n}(f) \downarrow 0$ as $n \uparrow \infty$. It is known (see, for example, [1, Ch. 1, Sect. 30]) that Fourier series of such functions converge everywhere expect maybe a countable set of points $x \equiv 0(\bmod 2 \pi)$; i. e., we have

$$
f(x)=\sum_{n=1}^{\infty}\left(a_{n}(f) \cos n x+b_{n}(f) \sin n x\right)
$$

almost everywhere in $\mathbb{R}$.

In the present paper, which is a continuation of the author's research $[5,6]$, we consider the problem of optimality of inequalities (3), (4), and (5) in terms of order equalities on the whole class $M_{p}(T)$ for $1<p<q<\infty$.

Theorem 1. Let $1<p<q<\infty, \sigma=1 / p-1 / q$, and $l, k \in \mathbb{N}$. A function $f \in M_{p}(\mathbb{T})$ belongs to $L_{q}(\mathbb{T})$ if and only if the condition $\Omega_{l}(f ; p ; \sigma ; q)<\infty$ holds. Moreover, the following order equalities hold:

$$
\begin{aligned}
& \|f\|_{q} \asymp \Omega_{l}(f ; p ; \sigma ; q) \equiv\left(\sum_{\nu=1}^{\infty} \nu^{q \sigma-1} \omega_{l}^{q}\left(f ; \frac{\pi}{\nu}\right)_{p}\right)^{1 / q} ; \\
& \omega_{k}\left(f ; \frac{\pi}{n}\right)_{q}+n^{\sigma} \omega_{l}\left(f ; \frac{\pi}{n}\right)_{p} \asymp\left(\sum_{\nu=n+1}^{\infty} \nu^{q \sigma-1} \omega_{l}^{q}\left(f ; \frac{\pi}{\nu}\right)_{p}\right)^{1 / q}, \quad n \in \mathbb{N}, \quad l \leq k ; \\
& \omega_{k}\left(f ; \frac{\pi}{n}\right)_{q} \asymp\left\{\left(\sum_{\nu=n+1}^{\infty} \nu^{q \sigma-1} \omega_{l}^{q}\left(f ; \frac{\pi}{\nu}\right)_{p}\right)^{1 / q}+\right. \\
& \left.+n^{-k}\left(\sum_{\nu=1}^{n} \nu^{q(k+\sigma)-1} \omega_{l}^{q}\left(f ; \frac{\pi}{\nu}\right)_{p}\right)^{1 / q}\right\}, \quad n \in \mathbb{N}, \quad l>k ; \\
& n^{-(l-\sigma)}\left(\sum_{\nu=1}^{n} \nu^{p(l-\sigma)-1} \omega_{k}^{p}\left(f ; \frac{\pi}{\nu}\right)_{q}\right)^{1 / p} \asymp\left(\sum_{\nu=n+1}^{\infty} \nu^{q \sigma-1} \omega_{l}^{q}\left(f ; \frac{\pi}{\nu}\right)_{p}\right)^{1 / q}, \quad n \in \mathbb{N}, \quad l \leq k ; \\
& \omega_{k}\left(f ; \frac{\pi}{n}\right)_{q}+n^{\sigma} \omega_{l}\left(f ; \frac{\pi}{n}\right)_{p} \asymp n^{-(l-\sigma)}\left(\sum_{\nu=1}^{n} \nu^{p(l-\sigma)-1} \omega_{k}^{p}\left(f ; \frac{\pi}{\nu}\right)_{q}\right)^{1 / p}, \quad n \in \mathbb{N}, \quad l \leq k .
\end{aligned}
$$

Remark 2. When evaluating from below in the order equality (7) the second term $n^{\sigma} \omega_{l}(f ; \pi / n)_{p}$ cannot be omitted in the general case, because there exists a function $g \in M_{p}(\mathbb{T})$ such that $n^{\sigma} \omega_{l}(g ; \pi / n)_{p} \neq O\left(\omega_{k}(g ; \pi / n)_{q}\right)$. The function $g \in M_{p}(\mathbb{T})$ is defined as follows (see [6, Sect. 3.1]): $g(x)=\sum_{n=1}^{\infty} a_{n} \cos n x$, where $a_{n}=a_{n}(p ; l)=n^{-(l+1-1 / p)}, n \in \mathbb{N}$, and the following order equalities hold: $E_{n-1}(g)_{p} \asymp n^{-l}, n \in \mathbb{N}$, and $\omega_{l}(g ; \pi / n)_{p} \asymp n^{-l}(\ln (e n))^{1 / p}, n \in \mathbb{N}$. Since $l>\sigma$, we have $g \in M_{q}(\mathbb{T})$; moreover,

$$
E_{n-1}(g)_{q} \asymp n^{-(l-\sigma)}, \quad n \in \mathbb{N} \Leftrightarrow \omega_{l}(g ; \pi / n)_{q} \asymp n^{-(l-\sigma)}, \quad n \in \mathbb{N} .
$$

Thus, in view of these order equalities, we have

$$
n^{\sigma} \omega_{l}(g ; \pi / n)_{p} \asymp n^{-(l-\sigma)}(\ln (e n))^{1 / p} \asymp \omega_{l}(g ; \pi / n)_{q}(\ln (e n))^{1 / p}, \quad n \in \mathbb{N} ;
$$

whence $n^{\sigma} \omega_{l}(g ; \pi / n)_{p} \neq O\left(\omega_{l}(g ; \pi / n)_{q}\right), n \in \mathbb{N}$, and a fortiori $n^{\sigma} \omega_{l}(g ; \pi / n)_{p} \neq O\left(\omega_{k}(g ; \pi / n)_{q}\right)$, $n \in \mathbb{N}$, in the case $k>l$. 
However, it can be omitted if the sequence $\left\{\omega_{l}(f ; \pi / n)_{p}\right\}_{n=1}^{\infty}$ satisfies Stechkin's $\left(S_{l}\right)$-condition $\left(\left\{\omega_{l}(f ; \pi / n)_{p}\right\}_{n=1}^{\infty} \in S_{l}\right)$ : there exists $\varepsilon \in(0, l)$ such that the sequence $\left\{n^{l-\varepsilon} \omega_{l}(f ; \pi / n)_{p}\right\}_{n=1}^{\infty}$ almost increases. This condition is equivalent to Bari's $\left(B_{l}^{(\alpha)}\right)$-condition for every fixed $\alpha \in[1, \infty)$ $\left(\left\{\omega_{l}(f ; \pi / n)_{p}\right\}_{n=1}^{\infty} \in B_{l}^{(\alpha)}\right)$ :

$$
n^{-l}\left(\sum_{\nu=1}^{n} \nu^{\alpha l-1} \omega_{l}^{\alpha}\left(f ; \frac{\pi}{\nu}\right)_{p}\right)^{1 / \alpha}=O\left(\omega_{l}\left(f ; \frac{\pi}{n}\right)_{p}\right), \quad n \in \mathbb{N},
$$

(see [6, Sect. 3.2)].

Theorem 2. Suppose that $1<p<q<\infty, f \in M_{p}(\mathbb{T}), \sigma=1 / p-1 / q, l, k \in \mathbb{N}, l \leq k$, and condition (1) holds. If $\left\{\omega_{l}(f ; \pi / n)_{p}\right\}_{n=1}^{\infty} \in S_{l}$, then the following order equality holds:

$$
\omega_{k}\left(f ; \frac{\pi}{n}\right)_{q} \asymp\left(\sum_{\nu=n+1}^{\infty} \nu^{q \sigma-1} \omega_{l}^{q}\left(f ; \frac{\pi}{\nu}\right)_{p}\right)^{1 / q}, \quad n \in \mathbb{N} .
$$

Remark 3. The condition $\left\{\omega_{l}(f ; \pi / n)_{p}\right\}_{n=1}^{\infty} \in S_{l}$ guarantees the validity of the estimate $n^{\sigma} \omega_{l}(f ; \pi / n)_{p} \leq C_{7}(k, l, p, q) \omega_{k}(f ; \pi / n)_{q}, n \in \mathbb{N}$, for all functions $f \in M_{q}(\mathbb{T})$, where $1<p<q<\infty$, $l, k \in \mathbb{N}$ (see the proof of Theorem 2, inequality (22)). In the case $l>k$, this estimate holds for $f \in M_{q}(\mathbb{T})$ without any conditions on the sequence $\left\{\omega_{l}(f ; \pi / n)_{p}\right\}_{n=1}^{\infty}$ (see the proof of Theorem 1 , inequality (18)).

Remark 4. In connection with Theorem 2, note also the following fact, which is an obvious corollary of the order equality (9) in Theorem 1: the order equality (11) is valid if and only if $\left\{\omega_{k}(f ; \pi / n)_{q}\right\}_{n=1}^{\infty} \in B_{l-\sigma}^{(p)}$. In addition, if $\left\{\omega_{l}(f ; \pi / n)_{p}\right\}_{n=1}^{\infty} \in B_{l}^{(p)} \Leftrightarrow\left\{\omega_{l}(f ; \pi / n)_{p}\right\}_{n=1}^{\infty} \in S_{l}$, then, in view of (11) and (9), we have $\left\{\omega_{k}(f ; \pi / n)_{q}\right\}_{n=1}^{\infty} \in B_{l-\sigma}^{(p)}$. On the other hand, in view of (11), the latter condition guarantees only that $\left\{\left(\sum_{\nu=n+1}^{\infty} \nu^{q \sigma-1} \omega_{l}^{q}(f ; \pi / \nu)_{p}\right)^{1 / q}\right\}_{n=1}^{\infty} \in B_{l-\sigma}^{(p)}$ but does not that $\left\{\omega_{l}(f ; \pi / n)_{p}\right\}_{n=1}^{\infty} \in B_{l}^{(p)}$.

P r o o f of Theorem 1. In the proof of Theorem 1, we will use neither estimates (2)-(5) from Theorems A and B nor the direct (see [6, inequality (0.3)]) and inverse (see [5, inequality (2)]) theorems of approximation theory for periodic functions in different metrics. We will use only certain known results that are characteristic of functions from the class $M_{p}(\mathbb{T})$ (the corresponding statements are gathered in [6, Sect. 1]). Since the auxiliary inequalities needed for the proofs of Theorems 1 and 2 were established by the author in [5,6], we will mostly refer to these papers instead of giving original references, which can be found in [5] and [6].

1) The first part of Theorem 1 and the order equality (6) were proved in [6, Sect. 2, the proof of statement (1) of Theorem 1]; more precisely, if $f \in M_{p}(\mathbb{T})$ and $\Omega_{l}(f ; p ; \sigma ; q)<\infty$, then $f \in L_{q}(\mathbb{T})$ and $\|f\|_{q} \leq C_{8}(l, p, q) \Omega_{l}(f ; p ; \sigma ; q)$ (the sufficiency); if $f \in M_{p}(\mathbb{T})$ belongs to $L_{q}(\mathbb{T})$, then $f \in M_{q}(\mathbb{T})$ and $\Omega_{l}(f ; p ; \sigma ; q) \leq C_{9}(l, p, q)\|f\|_{q}$ (the necessity). Moreover, in the proof of statement (1) of $\left[6\right.$, Theorem 1], we actually obtained the following estimates for a function $f \in M_{p}(\mathbb{T})$ under the condition $\Omega_{l}(f ; p ; \sigma ; q)<\infty$ (see also [5, Sect. 1]):

$$
C_{10}(p, q) E(f ; p ; \sigma ; q) \leq\|f\|_{q} \leq C_{11}(p, q) E(f ; p ; \sigma ; q),
$$

where

$$
E(f ; p ; \sigma ; q) \equiv\left(\sum_{\nu=1}^{\infty} \nu^{q \sigma-1} E_{\nu-1}^{q}(f)_{p}\right)^{1 / q} \asymp\left(\sum_{\nu=1}^{\infty} \nu^{q \sigma-1} \omega_{l}^{q}\left(f ; \frac{\pi}{\nu}\right)_{p}\right)^{1 / q} \equiv \Omega_{l}(f ; p ; \sigma ; q) .
$$


2) The upper estimate in (7): taking into account the inequality (see [6, Sect. 2, inequality (2.1)])

$$
n^{\sigma} \omega_{l}\left(f ; \frac{\pi}{n}\right)_{p} \leq C_{12}(l, p, q)\left(\sum_{\nu=n+1}^{\infty} \nu^{q \sigma-1} \omega_{l}^{q}\left(f ; \frac{\pi}{\nu}\right)_{p}\right)^{1 / q}, \quad n \in \mathbb{N}, \quad l \in \mathbb{N},
$$

in the estimation of $E_{n-1}(f)_{q}$ from above (see [6, Sect. 2, step The upper estimate in the proof of statement (2) of Theorem 1]), we obtain

$$
E_{n-1}(f)_{q} \leq C_{13}(l, p, q)\left(\sum_{\nu=n+1}^{\infty} \nu^{q \sigma-1} \omega_{l}^{q}\left(f ; \frac{\pi}{\nu}\right)_{p}\right)^{1 / q}, \quad n \in \mathbb{N}, \quad l \in \mathbb{N} .
$$

Further, using estimate (14) in the inequality (see [6, Sect. 1, Lemma 1, inequality (1.8)])

$$
\omega_{k}\left(f ; \frac{\pi}{n}\right)_{q} \leq C_{14}(k, p, q)\left\{E_{n}(f)_{q}+n^{\sigma} \omega_{k}\left(f ; \frac{\pi}{n}\right)_{p}\right\}, \quad n \in \mathbb{N}, \quad k \in \mathbb{N}
$$

and taking into account (13), we obtain for $l \leq k$

$$
\begin{gathered}
\omega_{k}\left(f ; \frac{\pi}{n}\right)_{q} \leq C_{14}(k, p, q)\left\{C_{13}(l, p, q)\left(\sum_{\nu=n+1}^{\infty} \nu^{q \sigma-1} \omega_{l}^{q}\left(f ; \frac{\pi}{\nu}\right)_{p}\right)^{1 / q}+2^{k-l} n^{\sigma} \omega_{l}\left(f ; \frac{\pi}{n}\right)_{p}\right\} \leq \\
\leq C_{15}(k, l, p, q)\left(\sum_{\nu=n+1}^{\infty} \nu^{q \sigma-1} \omega_{l}^{q}\left(f ; \frac{\pi}{\nu}\right)_{p}\right)^{1 / q}, \quad n \in \mathbb{N}
\end{gathered}
$$

where $C_{15}(k, l, p, q)=C_{14}(k, p, q)\left\{C_{13}(l, p, q)+2^{k-l} C_{12}(l, p, q)\right\}$; whence,

$$
\omega_{k}\left(f ; \frac{\pi}{n}\right)_{q}+n^{\sigma} \omega_{l}\left(f ; \frac{\pi}{n}\right)_{p} \leq\left(C_{15}(k, l, p, q)+C_{12}(l, p, q)\right)\left(\sum_{\nu=n+1}^{\infty} \nu^{q \sigma-1} \omega_{l}^{q}\left(f ; \frac{\pi}{\nu}\right)_{p}\right)^{1 / q}, \quad n \in \mathbb{N} .
$$

The lower estimate in (7): applying to the inequality (see [6, Sect. 2, step The lower estimate in the proof of statement (2) of Theorem 1])

$$
\begin{gathered}
\left(\sum_{\nu=n+1}^{\infty} \nu^{q \sigma-1} \omega_{l}^{q}\left(f ; \frac{\pi}{\nu}\right)_{p}\right)^{1 / q} \leq \\
\leq C_{16}(l, p, q)\left\{n^{\sigma-l}\left(\sum_{\nu=1}^{n} \nu^{p l-1} E_{\nu-1}^{p}(f)_{p}\right)^{1 / p}+\left(\sum_{\nu=n+1}^{\infty} \nu^{q \sigma-1} E_{\nu-1}^{q}(f)_{p}\right)^{1 / q}\right\}
\end{gathered}
$$

the lower estimate in the order equality (1.7) from [6, Sect. 1, Proposition 5]:

$$
\omega_{l}\left(f ; \frac{\pi}{n}\right)_{p} \asymp n^{-l}\left(\sum_{\nu=1}^{n} \nu^{p l-1} E_{\nu-1}^{p}(f)_{p}\right)^{1 / p}, \quad n \in \mathbb{N}, \quad l \in \mathbb{N},
$$

and inequality (10) from [5, Sect. 1]:

$$
\left(\sum_{\nu=n+1}^{\infty} \nu^{q \sigma-1} E_{\nu-1}^{q}(f)_{p}\right)^{1 / q} \leq C_{17}(k, p, q) \omega_{k}\left(f ; \frac{\pi}{n}\right)_{q}, \quad n \in \mathbb{N}, \quad k \in \mathbb{N},
$$

we obtain for arbitrary $l, k \in \mathbb{N}$

$$
\begin{gathered}
\left(\sum_{\nu=n+1}^{\infty} \nu^{q \sigma-1} \omega_{l}^{q}\left(f ; \frac{\pi}{\nu}\right)_{p}\right)^{1 / q} \leq C_{16}(l, p, q)\left\{C_{18}(l, p) n^{\sigma} \omega_{l}\left(f ; \frac{\pi}{n}\right)_{p}+\right. \\
\left.+C_{17}(k, p, q) \omega_{k}\left(f ; \frac{\pi}{n}\right)_{q}\right\} \leq C_{19}(k, l, p, q)\left\{\omega_{k}\left(f ; \frac{\pi}{n}\right)_{q}+n^{\sigma} \omega_{l}\left(f ; \frac{\pi}{n}\right)_{p}\right\}, \quad n \in \mathbb{N} .
\end{gathered}
$$


3) The upper estimate in (8): if $f \in M_{p}(\mathbb{T})$ and $\Omega_{l}(f ; p ; \sigma ; q)<\infty$, then $f \in L_{q}(\mathbb{T})$ and, consequently, $f \in M_{q}(\mathbb{T})$. By the upper estimate in (16) and inequality (14), we have

$$
\begin{gathered}
\omega_{k}\left(f ; \frac{\pi}{n}\right)_{q} \leq C_{20}(k, q) n^{-k}\left(\sum_{\nu=1}^{n} \nu^{q k-1} E_{\nu-1}^{q}(f)_{q}\right)^{1 / q} \leq \\
\leq C_{20}(k, q) C_{13}(l, p, q) n^{-k}\left(\sum_{\nu=1}^{n} \nu^{q k-1} \sum_{\mu=\nu+1}^{n} \mu^{q \sigma-1} \omega_{l}^{q}\left(f ; \frac{\pi}{\mu}\right)_{p}+\right. \\
\left.+\sum_{\nu=1}^{n} \nu^{q k-1} \sum_{\mu=n+1}^{\infty} \mu^{q \sigma-1} \omega_{l}^{q}\left(f ; \frac{\pi}{\mu}\right)_{p}\right)^{1 / q} \leq \\
\leq C_{21}(k, l, p, q) n^{-k}\left(\sum_{\mu=1}^{n} \mu^{q \sigma-1} \omega_{l}^{q}\left(f ; \frac{\pi}{\mu}\right)_{p} \sum_{\nu=1}^{\mu} \nu^{q k-1}+\sum_{\nu=1}^{n} \nu^{q k-1} \sum_{\mu=n+1}^{\infty} \mu^{q \sigma-1} \omega_{l}^{q}\left(f ; \frac{\pi}{\mu}\right)_{p}\right)^{1 / q} \leq \\
\leq C_{21}(k, l, p, q) n^{-k}\left(\sum_{\mu=1}^{n} \mu^{q(k+\sigma)-1} \omega_{l}^{q}\left(f ; \frac{\pi}{\mu}\right)_{p}+n^{q k} \sum_{\mu=n+1}^{\infty} \mu^{q \sigma-1} \omega_{l}^{q}\left(f ; \frac{\pi}{\mu}\right)_{p}\right)^{1 / q} \leq \\
\leq C_{21}(k, l, p, q)\left\{n^{-k}\left(\sum_{\mu=1}^{n} \mu^{q(k+\sigma)-1} \omega_{l}^{q}\left(f ; \frac{\pi}{\mu}\right)_{p}\right)^{1 / q}+\left(\sum_{\mu=n+1}^{\infty} \mu^{q \sigma-1} \omega_{l}^{q}\left(f ; \frac{\pi}{\mu}\right)_{p}\right)^{1 / q}\right\}, n \in \mathbb{N} .
\end{gathered}
$$

The lower estimate in (8): let us first prove that the following estimate holds for $l>k$ :

$$
n^{\sigma} \omega_{l}\left(f ; \frac{\pi}{n}\right)_{p} \leq C_{22}(k, l, p, q) \omega_{k}\left(f ; \frac{\pi}{n}\right)_{q}, \quad n \in \mathbb{N} .
$$

To this end, we will need the inequality (see [6, Sect. 2, inequality (2.6)]

$$
n^{\sigma} E_{n-1}(f)_{p} \leq C_{23}(k, p, q) \omega_{k}\left(f ; \frac{\pi}{n}\right)_{q}, \quad n \in \mathbb{N}, \quad k \in \mathbb{N} .
$$

Applying (19) in the upper estimate in (16) and taking into account the following known property of the modulus of smoothness: $\delta_{2}^{-k} \omega_{k}\left(f ; \delta_{2}\right)_{q} \leq 2^{k} \delta_{1}^{-k} \omega_{k}\left(f ; \delta_{1}\right)_{q}$ for $0<\delta_{1} \leq \delta_{2} \Leftrightarrow \nu^{k} \omega_{k}(f ; \pi / \nu)_{q} \leq$ $2^{k} n^{k} \omega_{k}(f ; \pi / n)_{q}$ for $1 \leq \nu \leq n$, we obtain

$$
\begin{gathered}
n^{\sigma} \omega_{l}\left(f ; \frac{\pi}{n}\right)_{p} \leq C_{20}(l, p) n^{\sigma-l}\left(\sum_{\nu=1}^{n} \nu^{p l-1} E_{\nu-1}^{p}(f)_{p}\right)^{1 / p} \leq \\
\leq C_{20}(l, p) C_{23}(k, p, q) n^{\sigma-l}\left(\sum_{\nu=1}^{n} \nu^{p l-1-p \sigma} \omega_{k}^{p}\left(f ; \frac{\pi}{\nu}\right)_{q}\right)^{1 / p} \leq \\
\leq C_{24}(k, l, p, q) n^{\sigma-l} 2^{k} n^{k} \omega_{k}\left(f ; \frac{\pi}{n}\right)_{q}\left(\sum_{\nu=1}^{n} \nu^{p(l-k-\sigma)-1}\right)^{1 / p} \leq C_{25}(k, l, p, q) \omega_{k}\left(f ; \frac{\pi}{n}\right)_{q},
\end{gathered}
$$

which implies the required estimate in (18).

Applying estimate (18) in inequality (17), we obtain the following upper estimate for the first term on the right-hand side of (8):

$$
\left(\sum_{\nu=n+1}^{\infty} \nu^{q \sigma-1} \omega_{l}^{q}\left(f ; \frac{\pi}{\nu}\right)_{p}\right)^{1 / q} \leq C_{19}(k, l, p, q)\left(1+C_{22}(k, l, p, q)\right) \omega_{k}\left(f ; \frac{\pi}{n}\right)_{q}, \quad n \in \mathbb{N} .
$$


The following estimate was obtained in $[5$, Sect. 1 , the proof of the lower estimate for the second term on the right-hand side of the order equality (7)]:

$$
n^{-k}\left(\sum_{\nu=1}^{n} \nu^{q(k+\sigma)-1} E_{\nu-1}^{q}(f)_{p}\right)^{1 / q} \leq C_{26}(k, p, q) \omega_{k}\left(f ; \frac{\pi}{n}\right)_{q}, \quad n \in \mathbb{N} .
$$

Hence, in view of the known order equality (see, for example, [4, Sect. 2, Remark 7, order equality (15)])

$$
\sum_{\nu=1}^{n} \nu^{\alpha \beta-1} E_{\nu-1}^{\alpha}(f)_{p} \asymp \sum_{\nu=1}^{n} \nu^{\alpha \beta-1} \omega_{l}^{\alpha}\left(f ; \frac{\pi}{\nu}\right)_{p},
$$

where $n \in \mathbb{N} \cup\{+\infty\}, 1 \leq \alpha<\infty$, and $0<\beta<l$, we obtain $(l>k \Rightarrow l>k+\sigma, \sigma \in(0,1))$

$$
\begin{gathered}
n^{-k}\left(\sum_{\nu=1}^{n} \nu^{q(k+\sigma)-1} \omega_{l}^{q}\left(f ; \frac{\pi}{\nu}\right)_{p}\right)^{1 / q} \asymp n^{-k}\left(\sum_{\nu=1}^{n} \nu^{q(k+\sigma)-1} E_{\nu-1}^{q}(f)_{p}\right)^{1 / q} \leq \\
\leq C_{26}(k, p, q) \omega_{k}\left(f ; \frac{\pi}{n}\right)_{q}, \quad n \in \mathbb{N} .
\end{gathered}
$$

The latter inequality implies the upper estimate for the second term on the right-hand side of (8).

4) The upper estimate in (9): the upper estimate in (12) implies the inequality

$$
E_{n-1}(f)_{q} \leq C_{27}(p, q)\left\{n^{\sigma} E_{n-1}(f)_{p}+\left(\sum_{\nu=n+1}^{\infty} \nu^{q \sigma-1} E_{\nu-1}^{q}(f)_{p}\right)^{1 / q}\right\}, \quad n \in \mathbb{N} .
$$

Indeed, applying (12) to the function $g_{n}(f ; x)=f(x)-S_{n}(f ; x)$, where $S_{n}(f ; x)$ is the partial sum of order $n \in \mathbb{N}$ of the Fourier series of the function $f \in M_{p}(T)$, and taking into account the estimate $E_{\nu-1}\left(g_{n}\right)_{p} \leq\left\|g_{n}\right\|_{p} \leq\left(1+C_{28}(p)\right) E_{n}(f)_{p}, \nu \in \mathbb{N}$, where $C_{28}(p)$ is the constant in the wellknown M. Riesz inequality $\left\|S_{n}(f ; \cdot)\right\|_{p} \leq C_{28}(p)\|f\|_{p}\left(1<p<\infty, f \in L_{p}(\mathbb{T})\right)$, and the equality $E_{\nu-1}\left(g_{n}\right)_{p}=E_{\nu-1}(f)_{p}, \nu \geq n+1, n \in \mathbb{Z}_{+}$, we obtain for $n \in \mathbb{N}$

$$
\begin{gathered}
E_{n}(f)_{q} \leq\left\|g_{n}(f ; \cdot)\right\|_{q} \leq C_{11}(p, q) E\left(g_{n} ; p ; \sigma ; q\right) \leq \\
\leq C_{11}(p, q)\left\{\left(\sum_{\nu=1}^{n+1} \nu^{q \sigma-1} E_{\nu-1}^{q}\left(g_{n}\right)_{p}\right)^{1 / q}+\left(\sum_{\nu=n+2}^{\infty} \nu^{q \sigma-1} E_{\nu-1}^{q}\left(g_{n}\right)_{p}\right)^{1 / q}\right\} \leq \\
\leq C_{11}(p, q)\left\{\left(1+C_{28}(p)\right) E_{n}(f)_{p}\left(\sum_{\nu=1}^{n+1} \nu^{q \sigma-1}\right)^{1 / q}+\left(\sum_{\nu=n+2}^{\infty} \nu^{q \sigma-1} E_{\nu-1}^{q}(f)_{p}\right)^{1 / q}\right\} \leq \\
\leq C_{11}(p, q)\left\{\left(1+C_{28}(p)\right) C_{29}(q, \sigma)(n+1)^{\sigma} E_{n}(f)_{p}+\left(\sum_{\nu=n+2}^{\infty} \nu^{q \sigma-1} E_{\nu-1}^{q}(f)_{p}\right)^{1 / q}\right\},
\end{gathered}
$$

and for $n=0$

$$
E_{0}(f)_{q} \leq\|f\|_{q} \leq C_{11}(p, q) E(f ; p ; \sigma ; q) \leq C_{11}(p, q)\left\{E_{0}(f)_{p}+\left(\sum_{\nu=2}^{\infty} \nu^{q \sigma-1} E_{\nu-1}^{q}(f)_{p}\right)^{1 / q}\right\} .
$$

Inequality (21) was used in [6, Sect. 2, step The upper estimate in the proof of statement (3) of Theorem 1] for obtaining the estimate

$$
n^{\sigma-l}\left(\sum_{\nu=1}^{n} \nu^{p(l-\sigma)-1} E_{\nu-1}^{p}(f)_{q}\right)^{1 / p} \leq C_{30}(l, p, q)\left(\sum_{\nu=n+1}^{\infty} \nu^{q \sigma-1} \omega_{l}^{q}\left(f ; \frac{\pi}{\nu}\right)_{p}\right)^{1 / q}, \quad n \in \mathbb{N}
$$


whence, by the order equality (20), we obtain the following upper estimate in (9):

$$
n^{-(l-\sigma)}\left(\sum_{\nu=1}^{n} \nu^{p(l-\sigma)-1} \omega_{k}^{p}\left(f ; \frac{\pi}{\nu}\right)_{q}\right)^{1 / p} \leq C_{31}(k, l, p, q)\left(\sum_{\nu=n+1}^{\infty} \nu^{q \sigma-1} \omega_{l}^{q}\left(f ; \frac{\pi}{\nu}\right)_{p}\right)^{1 / q}, \quad n \in \mathbb{N}, \quad l \leq k ;
$$

The lower estimate in (9): by inequality (17), we have for all $l, k \in \mathbb{N}$

$$
\left(\sum_{\nu=n+1}^{\infty} \nu^{q \sigma-1} \omega_{l}^{q}\left(f ; \frac{\pi}{\nu}\right)_{p}\right)^{1 / q} \leq C_{19}(k, l, p, q)\left\{\omega_{k}\left(f ; \frac{\pi}{n}\right)_{q}+n^{\sigma} \omega_{l}\left(f ; \frac{\pi}{n}\right)_{p}\right\}, \quad n \in \mathbb{N} .
$$

The upper estimate for the first term $\omega_{k}(f ; \pi / n)_{q}$ on the right-hand side is obvious, because, in view of the fact that $\omega_{k}(f ; \pi / n)_{q} \downarrow(n \uparrow)$, we have

$$
\begin{gathered}
n^{-(l-\sigma)}\left(\sum_{\nu=1}^{n} \nu^{p(l-\sigma)-1} \omega_{k}^{p}\left(f ; \frac{\pi}{\nu}\right)_{q}\right)^{1 / p} \geq \\
\geq n^{-(l-\sigma)} \omega_{k}\left(f ; \frac{\pi}{n}\right)_{q}\left(\sum_{\nu=1}^{n} \nu^{p(l-\sigma)-1}\right)^{1 / p} \geq C_{32}(l, p, q) \omega_{k}\left(f ; \frac{\pi}{n}\right)_{q} .
\end{gathered}
$$

The following upper estimate for the second term $n^{\sigma} \omega_{l}(f ; \pi / n)_{p}$ was established above (see the proof of the lower estimate in (8) at step 3):

$$
n^{\sigma} \omega_{l}\left(f ; \frac{\pi}{n}\right)_{p} \leq C_{20}(l, p) C_{23}(k, p, q) n^{\sigma-l}\left(\sum_{\nu=1}^{n} \nu^{p(l-\sigma)-1} \omega_{k}^{p}\left(f ; \frac{\pi}{\nu}\right)_{q}\right)^{1 / p} .
$$

Combining the obtained inequalities, we come to the required lower estimate in the order equality (9):

$$
\left(\sum_{\nu=n+1}^{\infty} \nu^{q \sigma-1} \omega_{l}^{q}\left(f ; \frac{\pi}{\nu}\right)_{p}\right)^{1 / q} \leq C_{33}(k, l, p, q) n^{-(l-\sigma)}\left(\sum_{\nu=1}^{n} \nu^{p(l-\sigma)-1} \omega_{k}^{p}\left(f ; \frac{\pi}{\nu}\right)_{q}\right)^{1 / p}, \quad n \in \mathbb{N} .
$$

5) The order equality (10) follows from (7) and (9). The proof of Theorem 1 is complete.

P r o o f of Theorem 2. The upper estimate in (11) was obtained at step 2 of the proof of Theorem 1 (see inequality (15)):

$$
\omega_{k}\left(f ; \frac{\pi}{n}\right)_{q} \leq C_{15}(k, l, p, q)\left(\sum_{\nu=n+1}^{\infty} \nu^{q \sigma-1} \omega_{l}^{q}\left(f ; \frac{\pi}{\nu}\right)_{p}\right)^{1 / q}, \quad n \in \mathbb{N} .
$$

To obtain the lower estimate in (11), we preliminarily prove that, if $\left\{\omega_{l}(f ; \pi / n)_{p}\right\}_{n=1}^{\infty} \in S_{l}$, then the following estimate holds for all $l, k \in \mathbb{N}$ :

$$
n^{\sigma} \omega_{l}\left(f ; \frac{\pi}{n}\right)_{p} \leq C_{34}(k, l, p, q) \omega_{k}\left(f ; \frac{\pi}{n}\right)_{q}, \quad n \in \mathbb{N} .
$$

Indeed, since

$$
\left\{\omega_{l}\left(f ; \frac{\pi}{n}\right)_{p}\right\}_{n=1}^{\infty} \in S_{l} \Leftrightarrow\left\{\omega_{l}\left(f ; \frac{\pi}{n}\right)_{p}\right\}_{n=1}^{\infty} \in B_{l}^{(p)} \Leftrightarrow\left\{E_{n-1}(f)_{p}\right\}_{n=1}^{\infty} \in B_{l}^{(p)}
$$

(see [6, Sect. 3.2)]), in view of [6, Sect. 2, inequality (2.8)] and [6, Introduction, inequality (0.4)], we obtain

$$
n^{\sigma} \omega_{l}\left(f ; \frac{\pi}{n}\right)_{p} \leq C_{35}(l, p, q) E_{n}(f)_{q} \leq C_{35}(l, p, q) C_{36}(k) \omega_{k}\left(f ; \frac{\pi}{n}\right)_{q}, \quad n \in \mathbb{N} .
$$


The required lower estimate in (11) follows from (17) and (22):

$$
\left(\sum_{\nu=n+1}^{\infty} \nu^{q \sigma-1} \omega_{l}^{q}\left(f ; \frac{\pi}{\nu}\right)_{p}\right)^{1 / q} \leq C_{19}(k, l, p, q)\left(1+C_{34}(k, l, p, q)\right) \omega_{k}\left(f ; \frac{\pi}{n}\right)_{q}, \quad n \in \mathbb{N} .
$$

The proof of Theorem 2 is complete.

Remark 5. By inequality (19), the upper estimate in the order equality (16) implies the inequality

$$
\omega_{l}\left(f ; \frac{\pi}{n}\right)_{p} \leq C_{37}(k, l, p, q) n^{-l}\left(\sum_{\nu=1}^{n} \nu^{p(l-\sigma)-1} \omega_{k}^{p}\left(f ; \frac{\pi}{\nu}\right)_{q}\right)^{1 / p}, \quad l, k, n \in \mathbb{N} .
$$

Inequality (23) (the case $l \leq k$ ) and inequality (18) (the case $l>k$ ) for functions $f \in$ $M_{q}(\mathbb{T}) \subset M_{p}(\mathbb{T})$ are inverse (in the sense of the upper estimate for $\omega_{l}(f ; \delta)_{p}$ in terms of $\left.\omega_{k}(f ; \delta)_{q}\right)$ to inequalities (3) and (4), respectively, which hold for all functions $f \in L_{q}(\mathbb{T})$ under the condition of convergence of series in (1). From inequalities (23) and (18), we can conclude that, in the passage from the class $M_{q}(\mathbb{T})$ to the class $M_{p}(\mathbb{T})$, where $p<q$, the smoothness of a function $f \in M_{q}(\mathbb{T})$ increases by a value not larger than $\sigma$ in the case $l \leq k$ (see [6, Sect. 3.3)], where the author considered the case $k=l$ and $\left.\omega_{l}(f ; \delta)_{q} \asymp \delta^{\alpha}, 0<\alpha \leq l, \delta \in(0, \pi]\right)$ and increases by a value not smaller than $\sigma$ in the case $l>k$.

\section{REFERENCES}

1. Bary N.K. A Treatise on Trigonometric Series. Vols. I, II. Oxford, New York: Pergamon Press, 1964, Vol. I, 533 p; Vol. II, 508 p. Original Russian text published in Trigonometricheskie ryady, Moscow: Fiz.-Mat. Giz. Publ., 1961, 936 p.

2. Gol'dman M.L. An imbedding criterion for different metrics for isotropic Besov spaces with arbitrary moduli of continuity. Proc. Steklov Inst. Math., 1994. No. 2. P. 155-181.

3. Il'yasov N. A. On the inequality between modulus of smoothness of various orders in different metrics. Math. Notes, 1991. Vol. 50, No. 2. P. 877-879. DOI: $10.1007 /$ BF01157580

4. Il'yasov N. A. On the direct theorem of approximation theory of periodic functions in different metrics. Proc. Steklov Inst. Math., 1997. Vol. 219. P. 215-230.

5. Il'yasov N.A. The inverse theorem in various metrics of approximation theory for periodic functions with monotone Fourier coefficients. Trudy Inst. Mat. i Mekh. UrO RAN [Proc. of Krasovskii Institute of Mathematics and Mechanics of the UB RAS], 2016. Vol. 22, No. 4. P. 153-162. (in Russian) DOI: $10.21538 / 0134-4889-2016-22-4-153-162$

6. Il'yasov N. A. The direct theorem of the theory of approximation of periodic functions with monotone Fourier coefficients in different metrics. Proc. Steklov Inst. Math., 2018. Vol. 303, Suppl. 1. P. S92-S106. DOI: $10.1134 /$ S0081543818090109

7. Kolyada V. I. On relations between moduli of continuity in different metrics. Proc. Steklov Inst. Math., 1989. Vol. 181. P. 127-148.

8. Timan M. F. Best approximation and modulus of smoothness of functions defined on the entire real axis. Izv. Vyssh. Ucheb. Zaved. Mat., 1961. No. 6. P. 108-120. (in Russian)

9. Timan M. F. Some embedding theorems for $L_{p}$-classes of functions. Dokl. Akad. Nauk SSSR,1970. Vol. 193, No. 6, P. 1251-1254. (in Russian)

10. Timan M.F. The imbedding of the $L_{p}^{(k)}$-classes of functions. Izv. Vyssh. Ucheb. Zaved. Mat., 1974. No. 10(149). P. 61-74. (in Russian)

11. Ul'yanov P. L. The imbedding of certain function classes $H_{p}^{\omega}$. Math. USSR-Izv., 1968. Vol. 2 , No. 3. P. 601-637.

12. Ul'yanov P. L. Imbedding theorems and relations between best approximations (moduli of continuity) in different metrics. Math. USSR-Sb., 1970. Vol. 10, No. 1. P. 103-126. 